\title{
Simvastatin improves the prognosis of endotoxin- induced disseminated intravascular coagulation by regulating the intestinal microenvironment
}

Min Xu

Institute of Haematology, Union Hospital, Tongji Medical College, Huazhong University of Science and Technology

Lili Luo

Institute of Haematology, Union Hospital, Tongji Medical College, Huazhong University of Science and Technology

\section{Mengyi Du}

Institute of Haematology, Union Hospital, Tongji Medical College, Huazhong University of Science and Technology

\section{Lu Tang}

Institute of Haematology, Union Hospital, Tongji Medical College, Huazhong University of Science and Technology

Jie Zhou

Institute of Haematology, Union Hospital, Tongji Medical College, Huazhong University of Science and Technology

\section{Yu Hu}

Institute of Haematology, Union Hospital, Tongji Medical College, Huazhong University of Science and Technology

Heng Mei ( $\sim$ hmei@hust.edu.cn )

Wuhan Union Hospital

\section{Research}

Keywords: Disseminated intravascular coagulation (DIC), simvastatin, inflammatory cytokines, intestinal permeability, intestinal microorganism

Posted Date: September 29th, 2020

DOl: https://doi.org/10.21203/rs.3.rs-76982/v1

License: (c) (1) This work is licensed under a Creative Commons Attribution 4.0 International License. Read Full License 


\section{Abstract}

Background: Disseminated intravascular coagulation (DIC) is characterized by extensive endothelial injury and coagulation activation that is primarily caused by infection and can be aggravated by the gut due to increased permeability and bacterial translocation. Studies have shown that statins play an important role in reducing inflammation, protecting the endothelium and improving coagulation. In addition, statins regulate tight junction (TJ) proteins and gut microbes. Therefore, we aimed to investigate whether simvastatin improves DIC prognosis by regulating the intestinal microenvironment.

Methods: Mice were administered $20 \mathrm{mg} / \mathrm{kg}$ simvastatin by gavage for 2 weeks and then intraperitoneally injected with $50 \mathrm{mg} / \mathrm{kg}$ endotoxin. Twelve hours later, cytokine release, coagulation dysfunction, multiple organ damage and survival were assessed. In addition, intestinal barrier and permeability and bacteria and bacteria translocation were evaluated.

Results: We found that the severity of endotoxin-induced DIC was significantly improved in simvastatinpretreated mice, who showed attenuated depletion of coagulation factors and platelets, decreased plasminogen activator inhibitor-1 (PAl-1) expression, reduced organ fibrin deposition and an improved survival rate. In addition, simvastatin reduced epithelial apoptosis, increased TJ gene expression, and upregulated antimicrobial peptides, lysozyme and mucins. Simvastatin-pretreated mice showed increased Lactobacillales counts, while the LPS group had increased numbers of Desulfovibrio and Mucispirillum, which produce harmful toxins and damage the intestinal epithelium and mucosa. Finally, with the decreased intestinal permeability in the simvastatin group, bacterial translocation in the organs and blood was significantly reduced, both in quantity and species.

Conclusions: Simvastatin improves DIC prognosis, and the intestinal microenvironment participates in this process.

\section{Background}

Disseminated intravascular coagulation (DIC), an imbalance in coagulation and fibrinolysis, is characterized by systemic endothelial damage and microthrombus formation, and if severe enough, DIC can induce multiple organ dysfunction syndrome (MODS) [1, 2]. Coagulation and inflammation are remarkably connected. A recent study reported that the overall mortality rate of severe sepsis is $21.5 \%$ and that the incidence of DIC in patients with sepsis is $50.9 \%$ [3]. The gradual progression of inflammation from systemic inflammatory response syndrome (SIRS) to severe sepsis parallels the increase in the prevalence of DIC and MODS [4]. Therefore, preventing progression to severe sepsis and reducing inflammatory factor levels are effective ways to restrain DIC and prevent the occurrence of MODS.

The intestine is mainly composed of epithelial cells connected via tight junctions (TJs), immune cells and intestinal microorganisms, which work together to maintain intestinal homeostasis [5]. Recent studies have revealed that changes in intestinal function are crucial for the development of sepsis and that 
increased intestinal permeability triggers a cascade of events resulting in increased bacterial growth and risk of sepsis [6]. Endotoxin-induced sepsis is related to gastrointestinal tract dysfunction and structural disorders. The most common complications are enhanced mucosal layer permeability, disturbed mucosal perfusion, development of tissue oedema, coagulation-associated local dysregulation, bacterial translocation and a shift in the gut microbiome [5]. For example, a recent study confirmed that patients with severe SIRS had significantly lower Bifidobacterium and Lactobacillus levels and higher levels of pathogenic bacteria than healthy volunteers [7]. A disturbance in intestinal flora and increased intestinal permeability can promote the entrance of harmful bacteria and bacterial products into local and distant spaces that are difficult to reach under normal circumstances, and potential outcomes are secondary infections, aggravated DIC and multiple organ dysfunction [6, 8]. In agreement with this, a correlation between gut barrier dysfunction and secondary lung injury has been reported [9]. In addition, not only do translocating bacteria and endotoxin from the gut lumen enter into systemic circulation but they also directly induce an immune response in the local gut-associated lymphoid tissue or draining lymph nodes, which results in significant systemic effects [9]. Therefore, improving intestinal microenvironment disorders is of great significance in reducing sepsis and DIC.

Statins, which are 3-hydroxy-3-methylglutaryl coenzyme A reductase inhibitors, reduce blood lipid levels and have pleiotropic effects, including improved endothelial dysfunction, reduced inflammation and inhibition of platelet activation $[10,11]$. Both clinical and animal studies have demonstrated that statins have beneficial effects in terms of both morbidity and mortality in severe sepsis or MODS $[12,13]$. Additionally, statins have been found to exert anticoagulant effects and promote fibrinolysis by reducing TF expression, increasing protein $C$ and thrombomodulin (TM) levels, and decreasing PAI-1 levels [11, 14, 15]. Considering the beneficial effects of statins on inflammation, coagulation and endothelial cells, statins might have significant value in improving DIC. A consensus has been reached on the antiinflammatory effects of statins in sepsis and the anti-thrombotic effects in cardiovascular diseases, but little is known about whether statins can improve DIC when serious infections are present.

In addition, studies have indicated that the distribution of TJ proteins in the membrane is alleviated by pro-inflammatory cytokines and nitric oxide (NO) $[16,17]$. Statins have the potential to reduce inflammation and regulate NO synthesis [18]. In agreement with this, a recent study confirmed that statins promote the expression of TJ proteins and alleviate kidney permeability [19]. Moreover, statins have been found to affect the diversity and composition of gut microbiota $[20,21]$. Several recent studies have also confirmed that the lipid-lowering effects of statins may be related to the gut $[21,22]$. Whether statins can improve intestinal microenvironment disorders and thus improve systemic inflammation and coagulation needs to be explored

Therefore, this study aimed to evaluate the effects of simvastatin in mice with endotoxin-induced DIC and to further explore potential changes in the gut.

\section{Materials And Methods}


Male C57BL/6J mice aged eight weeks were purchased from Beijing HFK Bio-Technology Co., Ltd. and housed at the SPF facility of Huazhong University of Science and Technology. All animal care and operations were performed in accordance with national and EU guidelines for the handling and use of experimental animals. Animal experiments were approved by the Animal Experimentation Ethics Committee of Huazhong University of Science and Technology (reference number 2266).

\section{Establishment of the animal model}

After a one-week adaptation period, the mice were randomly divided into three groups: control, LPS and LPS + SIM groups. Mice in the LPS + SIM group were given simvastatin $(20 \mathrm{mg} / \mathrm{kg}$, MedChem Express, NJ, USA) daily by gavage at noon for 2 weeks [22], and the other two groups were administered sterile water. Two weeks later, mice in the LPS and LPS + SIM groups were intraperitoneally injected with $50 \mathrm{mg} / \mathrm{kg}$ LPS (Escherichia coli, 055: B5, Sigma, St Louis, MO, USA) to establish the DIC model [2], and the control mice were injected with normal saline. Saline $(0.5 \mathrm{~mL})$ was administered every $6 \mathrm{~h}$. The animals in each group were sacrificed at $12 \mathrm{~h}$ after the LPS injection, and a few mice died at $6 \mathrm{~h}$. Blood was drawn from the inferior cava of anaesthetized mice ( $1 \%$ pentobarbital sodium, $50 \mathrm{mg} / \mathrm{kg}$, i.p.) and anticoagulated with ethylenediaminetetraacetic acid (EDTA, $10 \mathrm{mM}$ ) or citrate (final concentration, 3.2\%). Samples were centrifuged at $3000 \times \mathrm{g}$ for $20 \mathrm{~min}$ at $4{ }^{\circ} \mathrm{C}$, and then, the supernatant was frozen at $-80{ }^{\circ} \mathrm{C}$ until analysis. Tissues were fixed or stored at $-80^{\circ} \mathrm{C}$ for histological examination or RNA extraction. Fresh stool samples were collected from the caecum, snap-frozen and stored at $-80{ }^{\circ} \mathrm{C}$ for subsequent analysis.

\section{Blood Assays}

DIC-related markers in plasma, including prothrombin time (PT), activated partial thromboplastin time (APTT), fibrinogen, fibrin/fibrinogen degradation product (FDP) levels and antithrombin III (ATIII) activity, were measured on an automated STA-R Evolution Analyzer (Stago, France). The platelet count (PLT) was determined on an automated animal blood counter (PE-6800VET, PROKAN Co., Ltd., Shenzhen, China). Serum levels of blood urea nitrogen (BUN), creatinine (CR), aspartate aminotransferase (AST) and alanine aminotransferase (ALT) were determined with an Automated Chemistry Analyzer (Chemray-240, Rayto, Shenzhen, China). Plasma concentrations of the cytokines interleukin (IL)-6, IL-10, monocyte chemoattractant protein-1 (MCP-1), interferon- $\gamma$ (IFN- $\gamma$ ), and tumour necrosis factor- $a$ (TNF- $a$ ) were determined with a Mouse Inflammation Cytometric Bead Array (CBA) kit (No. 552364, BD, Pharmingen, San Jose, CA) according to the manufacturer's instructions. Plasma levels of mouse IL-1 $\beta$ and IL-18 were measured using enzyme-linked immunosorbent assay (ELISA) kits obtained from BD Pharmingen (No. 560232; No. 216165). We also measured various haemostatic endothelial molecular markers, including soluble thrombomodulin (sTM), thrombin-antithrombin complex (TAT) and PAI-1, using appropriate ELISA kits (Abcam).

\section{Histological Examination}


Ileum tissue samples were immediately washed with cold $0.9 \%$ saline solution and fixed in $4 \%$ paraformaldehyde for at least $24 \mathrm{~h}$. The fixed samples were embedded in paraffin and sectioned. The sections were deparaffinized, dehydrated and stained with haematoxylin and eosin for histological assessment of the intestinal mucosa. Changes in the intestinal mucosa were observed with an optical microscope (Leica, Germany).

\section{Immunohistochemistry}

Tissues samples were fixed in $4 \%$ paraformaldehyde and embedded in paraffin. Then, the sections were immunostained with antibodies against fibrinogen (1:20000 dilution, Abcam, Cambridge, UK), TF (1:400 dilution, Abcam, Cambridge, UK) and Ly-6G (1:200 dilution, BD Pharmingen) according to the manufacturers' protocols.

\section{Real-time Polymerase Chain Reaction}

TRIzol reagent (Takara, Dalian, China) was used to isolate total RNA from tissues and cells according to the manufacturer's instructions. cDNA was generated using a PrimeScript RT reagent kit (Takara, Dalian, China), and qRT-PCR was performed using a SYBR Green RT-PCR kit (Takara). GAPDH was used as an internal control, and the primers are shown in Table S1. All PCRs were run on an ABI 7500 FAST RealTime PCR System (Applied Biosystems), and each sample was tested in triplicate. Relative expression was calculated using the comparative $2^{-\rho \rho C T}$ method.

\section{Western blot analysis}

Frozen ileum segments were homogenized, and the supernatant was collected. The total protein concentration was measured via a BCA protein assay. A total of 20-30 $\mu \mathrm{g}$ of ileum tissue lysate was separated on $10 \%$ SDS-polyacrylamide gels and transferred to polyvinylidene fluoride membranes. After being blocked, the membranes were incubated overnight at $4{ }^{\circ} \mathrm{C}$ with primary antibodies against the following proteins: Bax (1:6000), Bcl-2 (1:1000), and caspase3 (1:1000) (Proteintech Group, Chicago, IL, USA). The membranes were washed three times and incubated with horseradish peroxidase-conjugated goat anti-rabbit secondary antibody (1:1000, Proteintech Group, Chicago, IL, USA) for 60 min at room temperature. Finally, the pixel density was detected with a chemiluminescence system (Pierce, Rockford, IL).

\section{In Vivo Intestinal Permeability Assay}

FITC-D4000 (FD-4, Sigma-Aldrich, St. Louis, MO) dissolved in saline to $50 \mathrm{mg} / \mathrm{ml}$ was given to the mice via gavage at a dose of $600 \mathrm{mg} / \mathrm{kg}$. One hour after gavage, blood was collected from anaesthetized mice and centrifuged at $12,000 \mathrm{rpm}$ at $4{ }^{\circ} \mathrm{C}$ for $10 \mathrm{~min}$ [23]. Fifty microliters of plasma was diluted $1: 2$ with phosphate-buffered saline (PBS, pH 7.4). The concentration of FD-4 was measured using a MultiDetection Microplate Reader (Synergy TM HT, Bio-Tek, Vermont, US) and KC4 software at an excitation wavelength of $493 \mathrm{~nm}$ and an emission wavelength of $518.5 \mathrm{~nm}$. Standards (range, 1000-7.8125 ng/ml) were obtained by diluting the FD-4 gavage stock solution with plasma from control animals. 


\section{Bacteriological Analysis}

Bacterial translocation was determined by culturing homogenized mesenteric lymph nodes (MLNs), lungs, liver, spleen, kidney, blood and peritoneal fluid (PLF). PLF was collected after rinsing the abdomen with $1 \mathrm{~mL}$ of isotonic saline, allowing the saline to remain within the peritoneal cavity for at least 30 seconds. Tissue samples were aseptically removed at $12 \mathrm{~h}$ after LPS administration and weighed and homogenized in PBS to achieve a $50 \mathrm{mg} / \mathrm{mL}$ concentration, and then, $0.1 \mathrm{~mL}$ of the samples were inoculated onto a Columbia Nutrient Agar plate with $5 \%$ sheep blood to grow total bacteria. Plates were cultured anaerobically in a $37^{\circ} \mathrm{C}$ incubator for $48 \mathrm{~h}$, and colonies were counted. Bacterial numbers are expressed as colony forming units per gram of tissues and per millilitre of blood or fluid.

\section{6s RDNA Sequencing}

Frozen faeces samples and lung samples were used to determine the gut microbiome profile. Genomic DNA was isolated using a PowerSoil@ DNA Isolation Kit (MO BIO Laboratories Inc., Carlsbad, CA, USA) according to the manufacturer's protocol. The high-throughput sequencing library was constructed and sequenced using the Illumina MiSeq platform by GENEWIZ (Suzhou, China). With 30-50 ng DNA as a template, a series of PCR primers designed by Jin Weizhi were employed to amplify prokaryotic $16 \mathrm{~S}$ rDNA, including the two highly variable regions V3 and V4, using a MetaVx Library Preparation kit (GENEWIZ).

Sequences longer than $200 \mathrm{bp}$ were retained, and sequence clustering was performed using VSEARCH (1.9.6) at the $97 \%$ similarity level [24]. The most abundant sequence in each operational taxonomic unit (OTU) was selected, and the Shannon index was calculated by random sampling of sample sequences. Based on the Bray-Curtis inter-sample distance matrix, the principal coordinate analysis (PCoA) was visualized to show the $\beta$ diversity. The abundance data for each sample were normalized and log transformed. The differences in microbial composition among different samples were assessed by analysis of similarities (ANOSIM).

\section{Cell Culture}

Human epithelial colorectal adenocarcinoma (Caco-2) cells were cultured in Dulbecco's modified Eagle's medium/high glucose (DMEM, Gibco) supplemented with $10 \%$ foetal bovine serum (FBS), penicillin and streptomycin. The cells were maintained in a $5 \% \mathrm{CO}_{2}$ incubator at $37^{\circ} \mathrm{C}$, and the culture medium was changed every 1 to 2 days. Trypsinized cells were added to 6-well tissue culture-treated plates (2000 $\mu$; Costar, Corning) at $2 \times 10^{5} \mathrm{cells} / \mathrm{cm}^{2}$. After 14 days, a confluent cell monolayer was obtained. The cells were stimulated with different concentrations of $\operatorname{simvastatin}(0,500 \mathrm{nM}, 1 \mu \mathrm{M}, 10 \mu \mathrm{M}$, or $20 \mu \mathrm{M})$ for $60 \mathrm{~h}$ and then co-treated with LPS $(20 \mu \mathrm{g} / \mathrm{mL})$ for $12 \mathrm{~h}$. Finally, the cells were collected for qPCR experiments.

\section{Statistical analysis}


All data in this study are presented as the mean \pm SEM, and $\mathrm{P}<0.05$ was considered to indicate statistical significance in all experiments. If the data had a normal distribution and homogeneity of variance, oneway ANOVA followed by Newman-Keuls post hoc test or Dunnett's $t$ test for multiple group comparisons was performed. Otherwise, a Kruskal-Wallis $H$ test was used. For comparisons of two groups, a $t$-test was used. Survival was analysed using a log rank test. Bacteriologic culture results were analysed with Fisher's exact test. All statistical analyses were performed using SPSS (version 19.0) or Prism 5 (GraphPad, La Jolla, CA, USA) software, and the figures were generated with Photoshop.

\section{Results}

\subsection{Simvastatin attenuates LPS-induced systemic inflammatory responses and organ failure and extends survival}

Plasma pro-inflammatory and anti-inflammatory factor levels, including IL-1 $\beta$, IL-6, and TNF-a, MCP-1, IFN- $\gamma$, IL-18 and IL-10, increased significantly in the LPS group. Simvastatin pretreatment decreased plasma pro-inflammatory cytokine levels and increased IL-10 levels compared with LPS alone (Fig. 1-A). This phenomenon was verified in lung tissue at the mRNA level (Fig. 1-B). The LPS group had a large number of infiltrating granulocytes in the lungs, which was significantly improved in the simvastatinpretreated group (Fig. 1-C).

Liver and kidney function was assessed to assess organ damage. Mice treated with simvastatin had significantly reduced serum ALT $(P<0.05)$, AST $(P<0.01)$ and CR $(P<0.01)$ levels compared with levels in the LPS group (Fig. 1-D). However, there was no significant difference in BUN between the two groups. Previous studies showed that a high dose of statins can cause abnormal liver function, but no significant difference in organ damage was detected between mice treated with simvastatin alone for 14 days (SIM group) and control mice (Fig. 1-D).

No deaths occurred in the control group. The survival curves for mice in the LPS group sharply declined, and all mice died within $20 \mathrm{~h}$. However, in the simvastatin-treated group, the survival rate increased by $20 \%$ at $48 \mathrm{~h}$ and $13.3 \%$ at $72 \mathrm{~h}(\mathrm{P}<0.001$, Fig. 2$)$. Continued observations revealed that the two surviving mice gradually recovered their appetite and activity from day 6 .

\subsection{Simvastatin improves blood coagulation disorders and fibrin deposition}

In this study, prophylactic administration of simvastatin helped shorten the prolongation of PT and APTT. The PLT, fibrinogen levels and ATIII dropped sharply at $12 \mathrm{~h}$ after the injection of endotoxin, and these decreases were significantly alleviated in the simvastatin group. After endotoxin injection, the PLT was $205 \pm 28 \times 10^{9} / \mathrm{L}$ in the LPS group and $305 \pm 44 \times 10^{9} / \mathrm{L}$ in the LPS $+S I M$ group, the corresponding fibrinogen levels were $1.6 \pm 0.2 \mathrm{~g} / \mathrm{L}$ and $2.5 \pm 0.3 \mathrm{~g} / \mathrm{L}(P<0.01)$, and the corresponding ATIII levels were 44 $\pm 2.5 \%$ and $54 \pm 1.6 \%$. PAl- 1 increased significantly after endotoxin administration $(161 \pm 25 \mathrm{ng} / \mathrm{mL})$, but this increase was attenuated in the simvastatin group $(95 \pm 6.4 \mathrm{ng} / \mathrm{mL})$. In addition, plasma TAT and TM 
showed a slight decrease in the LPS+SIM group compared to the LPS group, but the differences were not significant. FDP levels were not significantly different between the two groups (Fig. 3-A).

Histologic studies showed elevated TF levels in lung tissue in the LPS group compared with the LPS+SIM group (Fig. 3-B). Additionally, more extensive fibrin deposition was observed in various organs at $12 \mathrm{~h}$ after endotoxin administration, and fibrin deposition was most pronounced in small and mid-sized vessels or vasculature. Notably, simvastatin treatment significantly inhibited thrombus deposition in those organs (Fig. 3-C). These observations clearly suggest that simvastatin pretreatment during endotoxin exposure contributes to prevention of DIC development.

\subsection{Simvastatin attenuates intestinal damage and apoptosis}

As shown in Figure 4-A, the intestinal mucosa of mice treated with saline was intact, with well-ordered villi. However, in the LPS group, the intestinal villi were disrupted, atrophic or even ruptured at locations of necrotic epithelial cells, and the mucosa was oedematous. The damage was significantly alleviated in the simvastatin-pretreated group; although the small intestinal villi were swollen and deformed, there was no fracture in the villi barrier (Fig. 4-A).

Decreased gut epithelial apoptosis is associated with an improved survival rate in sepsis [25]. In our study, simvastatin reversed the LPS-induced upregulation of caspase3 and downregulation of $\mathrm{Bcl}-2$ at the protein level, and these results were verified at the mRNA level (Fig. 4-B, C).

\subsection{Simvastatin-treated mice showed different gut microbiota compositions}

The 16S rDNA sequence was used to explore the microbiota composition in the three groups. The ANOSIM of PCOA matrix scores indicated a significant separation in the microbiota composition in the three groups $(P<0.05$; Fig. $5-A)$. LPS treatment resulted in an increase in community diversity, as shown by the Shannon index, and the statin decreased community diversity, but there were no significant differences among groups (Fig. 5-C). The gut microbiota compositions at the phylum level are shown in Figure 5-B. In both of the treated groups, the top two phyla were Firmicutes and Bacteroidetes. Compared to the control group, the LPS group showed an increased abundance of Firmicutes (65.6\% vs. $45.5 \%$ ) and Proteobacteria ( $10.6 \%$ vs. $2.8 \%$ ) and a decreased abundance of Bacteroidetes ( $20.5 \%$ vs. $45.7 \%$ ). In contrast, the simvastatin-treated group showed decreased Firmicutes $(60.5 \%)$ and Proteobacteria (3.5\%) and increased Bacteroidetes (31.7\%) compared to the LPS group and was more similar to the control group. These data suggest that the simvastatin-treated mice had a different gut microbiota composition than the LPS-treated mice but a composition similar to the control mice (Fig. 5-B).

Distinctive gut microbiota compositions in the indicated groups were also identified by linear discriminant analysis. Endotoxin treatment induced a marked increase in the abundance of harmful bacteria, including Desulfovibrio $(P<0.05)$ in the Proteobacteria phylum and Mucispirillum $(P<0.05)$ in the Deferribacteres phylum. Desulfovibrio can produce hydrogen sulfide to destroy intestinal epithelial cells, and Mucispirillum can directly decompose mucus [26, 27]. LPS also increased the abundance of 
Ruminococcaceae (Firmicutes phylum). Conversely, statin therapy triggered a considerable enrichment of bacteria in the class Bacilli; however, this expansion was not class-wide but rather was due to the expansion of Lactobacillaceae (Fig. 5-D, E).

\subsection{Simvastatin alters intestinal barrier function and gut permeability}

In our study, the mRNA levels of ZO-1 and JAM did not change significantly in the LPS group but were markedly increased by simvastatin treatment (Fig. 6-A). However, occludin and claudin-4 levels were sharply decreased in both the LPS and LPS+SIM groups. In Caco-2 cells, ZO-1, JAM-A, occludin and claudin-4 levels increased in a concentration-dependent manner in the simvastatin-treated group (Fig. 6B), which was inconsistent with the in vivo experiments. We hypothesize that the intestine is a more complex system than Caco-2 cells, which can regulate TJ proteins through complex mechanisms. However, these results confirmed that statins alter the expression of TJ proteins.

Chemical barriers, including lysozyme, digestive enzymes, mucopolysaccharide and antimicrobial peptides, are also important components of the intestinal mucosal barrier. As expected, Reg3b, Defb-1 and angiogerin- 1 were significantly downregulated at $12 \mathrm{~h}$ of endotoxin treatment, but these effects were significantly reversed in the simvastatin pretreatment group. However, Reg3 $\mathrm{g}$ increased 4 -fold in both the LPS and LPS+SIM group (Fig. 6-C).

Mucus is another barrier that can protect epithelial cells from digestive enzymes [28]. Core 3 $31,3-\mathrm{N}-$ acetylglucosaminyltransferase (C3 gnt) is responsible for glycosylation of intestinal mucins, and muc2 can disassociate pathogenic and commensal bacteria [28, 29]. As expected, the mRNA levels of muc2 and C3 gnt were significantly decreased in the LPS group but increased in the simvastatin group. Additionally, the expression levels of intestinal alkaline phosphatase (IAP, detoxifies bacterial LPS) were increased in the simvastatin treatment group. However, no significant changes in the levels of lysozyme (protects against bacterial infection) were observed (Fig. 6-D).

Intestinal inflammation is also a factor affecting intestinal permeability. The mRNA levels of inflammatory cytokines in the intestine were detected. Unexpectedly, simvastatin administration did not decrease the LPS-induced increases in IL-1 $\beta$, MCP-1 and IL- 6 mRNA levels. TNF- $\alpha$ mRNA levels were significantly upregulated and the anti-inflammatory cytokine IL-10 was downregulated by simvastatin administration compared with LPS alone (Fig. 6-E). This was inconsistent with the regulatory effect of simvastatin in the lung tissue (Fig. 1-B), which may be due to the reduction in intestinal permeability caused by simvastatin, resulting in accumulation of intestinal toxins, which in turn activates the inflammatory response.

As shown in Figure 6-F, intestinal permeability was measured at $0 \mathrm{~h}, 6 \mathrm{~h}$ and $12 \mathrm{~h}$ after LPS injection. There was no difference in intestinal permeability between the LPS group and LPS+SIM group at baseline; at $6 \mathrm{~h}$, the permeability was significantly lower in the LPS+SIM group than in the LPS group, but this difference was not obvious at $12 \mathrm{~h}$. 


\section{3-6 Simvastatin inhibits translocation of intestinal flora}

To further explore whether simvastatin pretreatment reduced bacterial translocation, we adopted bacterial cultures in organs and blood. We found that the positive rate of bacterial culture in the LPS group was higher than that in the LPS + SIM group, especially in the liver, kidney and blood (Fig. 7-A). We further counted the number of positive bacterial colonies and found that in the liver, kidney, lung, spleen and blood, the content of translocation bacteria in the LPS group was much higher than that in the LPS + SIM group (Fig. 7, B1-5). However, no difference was found in colony units between MLNs and FLF, which are outposts for bacterial translocation (Fig. 7, B6-7).

In addition, 16 sDNA sequencing revealed that the lung tissue microbial amplification success rate reached $100 \%$ in the LPS group, higher than that in the LPS + SIM group (62.5\%) and consistent with the bacterial culture results (Fig. 7-C). The LPS and LPS + SIM groups showed different distributions of translocated microbes in the lung tissues, with species and number being more abundant in the LPS group (Fig. 7-D, E, F). The significantly increased flora in the LPS group included Escherichia-Shigella, Helicobacter, Faecalibacterium, Lachnospiraceae, Bacteroides and Bifidobacterium, while Lactococcus and Streptococcus were higher in the LPS+SIM group (Fig. 7-G, H).

\section{Discussion}

In this study, we systematically explored the effects of statins on endotoxin-induced DIC and found that LPS alone induced more serious DIC with more pronounced coagulation factor depletion and fibrin deposition, while simvastatin attenuated DIC progression by enhancing anticoagulant levels and fibrinolysis. These findings might explain the alleviated organ microthrombosis and tissue damage, which contributed to prevention of multiple organ failure and death in the simvastatin group. In addition, we found that the intestine plays an important role in this process. Simvastatin pretreatment reduced the intestinal epithelial damage and apoptosis and altered the intestinal microbial composition, with increased Lactobacillales in the LPS + SIM group and increased Desulfovibrio and Mucispirillum in the LPS group. Additionally, simvastatin treatment increased TJ protein and antibacterial peptide levels, and therefore, reduced intestinal permeability. With the above changes, the translocation of intestinal flora in organs and blood were reduced, avoiding the aggravation of primary infection and causing secondary infection due to intestinal disorders. Therefore, in addition to the known direct regulation effects of statins, they also indirectly improve systemic inflammation through the intestines, thereby regulating coagulation and the prognosis of DIC.

Activation of the coagulation system plays a pivotal role in the pathogenesis of DIC and the development of multiple organ failure. Consistent with previous studies, simvastatin pretreatment reduced inflammation and improved the coagulation disorder and mortality [13]. TF levels have been shown to increase 125-fold in blood monocytes in healthy humans after the administration of endotoxin at a low dose [30], but a consensus has been reached on the ability of statins to reduce TF expression [15]. Fibrinogen regulation by statins is controversial, and discrepancies may be related to different types of 
statins and laboratory tests. In our experiments, simvastatin pretreatment alleviated LPS-induced fibrinogen consumption. Increased plasma TM levels is a marker of endothelial damage, and statins protect endothelial cells and maintain anticoagulant properties by upregulating TM gene expression and reducing release [31]. In addition, consistent with the findings of a recent meta-analysis, statin therapy significantly lowered the plasma PAI-1 concentration [14]. The pronounced improvement in the imbalance between coagulation and fibrinolysis in the SIM group was associated with less severe tissue damage and a lower mortality rate.

Long-term studies have shown that the gut plays an important role in sepsis and MODS progression[5, 6]. Gut epithelial apoptosis is increased in sepsis, and overexpression of the anti-apoptotic protein Bcl-2 can improve survival in murine sepsis models [25]. In our experiments, statins alleviated apoptosis in the intestine, accompanied by decreased caspase 3 and increased Bcl-2 expression.

A biological barrier composed of gut microbes is essential to maintain gut homeostasis. Consistent with a recent study, we found that the Bacteroidetes/Firmicutes ratio was decreased in the LPS group but restored in the simvastatin group [20]. In the Linear discriminant analysis Effect Size (LEfSe), the simvastatin-treated group showed an increased abundance of beneficial bacterium in the class Bacilli. This expansion was not class-wide but was due to an increase in the order Lactobacillaceae. Studies have shown that exogenous Lactobacillus supplementation is associated with a lower incidence of sepsis in newborns and intensive care unit patients with ventilator-associated pneumonia [32, 33]. Bacteria in the genus Desulfovibrio, the major genus in the Desulfovibrionaceae family, generate hydrogen sulfide, a genotoxin and mucosal barrier-breaker, and most Desulfovibrionaceae members are reported to produce LPS and cause low-grade and chronic inflammation in obese subjects [26]. The population of these bacteria was increased in the LPS group. The further increase in Deferribacteraceae in the LPS group largely accounted for the expansion of Mucispirillum, which is known as a mucin degrader and is associated with early disruption of the colonic surface mucus layer [27]. Therefore, these differences in gut microbiota might be important reasons why statins improve the sepsis and DIC prognosis.

Mucosal barrier dysfunction is the most frequent complication within the gastrointestinal tract during sepsis [5]. TJs between intestinal epithelial cells form a barrier that selectively controls the transport of intestinal contents to the blood and prevents entry of antigens, microorganisms, and their toxins into the body. Disruption of the TJ barrier can result in increased intestinal permeability and promote bacterial stasis, bacterial overgrowth, and bacterial translocation [6, 8]. The concept that LPS induces inflammatory cytokine release, which leads to increased NO production and gut injury and contributes to the loss of gut barrier function, is not novel $[16,17]$. Signalling pathways, such as the myosin light chain kinase (MLCK), protein kinase $\mathrm{C}$ and Rho GTPase pathways, are also involved in the regulation of TJs [19, $34,35]$. Statins increase the expression of ZO-1 and JAM, possibly related to regulation of the balance between eNOS and iNOS and related signalling pathways [18]. However, occludin and claudin-4 levels were markedly decreased in both the LPS and simvastatin groups at $12 \mathrm{~h}$. We speculate that the accumulation of harmful substances in the intestine led to an increase in intestinal inflammation in the 
simvastatin-treated group, which in turn inhibited the expression of TJ proteins through activation of MLCK [5]. Notably, several TJ proteins were upregulated in simvastatin-treated Caco-2 cells. Alternatively, changes in gut microbes and specific activation of the immune system are involved in this process. For example, the probiotics Streptococcus thermophilus and Lactobacillus acidophilus can protect against TJ disruption via Rho family GTPases [36].

The chemical barrier is another important player in barrier function. C3gnt is responsible for glycosylation of intestinal mucins, thus providing an important source of growth substrates for intestinal bacteria [29]. Muc2 can disassociate pathogenic and commensal bacteria. Muc2-/- mice are highly susceptible to sepsis $[6,28]$, and lysozyme was shown to complex with high affinity to bacterial LPS and inhibit its biological activity [37]. Additionally, IAP is a gut mucosal defence factor known to dephosphorylate LPS [38]. The antibacterial peptides angiogerin-1, Defb-1, Reg3b and Reg3 g were reported to have broadspectrum antibacterial activity and quickly kill targets to inhibit bacterial translocation and intestinal inflammation [39].

Inflammation is another factor affecting intestinal barrier function. However, we found that simvastatin did not reduce intestinal inflammation, which is contrary to the anti-inflammatory effect of statins on organs such as the lungs. This result is unique, a previous study found that statin pretreatment downregulated inflammatory genes in the liver and increased intestinal inflammation[40]. We hypothesize that simvastatin pretreatment reduces intestinal permeability, which suppresses the movement of intestinal toxic exudate that accumulates in the intestine and promotes upregulation of intestinal inflammatory genes. In addition, the immune system can be triggered by gut microbiota [41]. Ligation to bacterial receptors stimulates central signalling cascades (e.g., NF-KB pathways), resulting in an immunological response and thereby regulating inflammation.

Simvastatin reduces intestinal permeability, which leads to reduced translocation of intestinal flora. In sepsis, the gut is severely damaged and the levels of harmful substances and microorganisms increase, which aggravates the primary infection and leads to secondary infections[8]. A recent study showed that increased intestinal permeability triggers a cascade of events that result in increased bacterial growth, bacterial translocation and risk of sepsis [6]. On the one hand, simvastatin improves systemic inflammation through direct anti-inflammatory and protective endothelial effects; on the other hand, it inhibits the translocation of harmful intestinal flora and toxins to the blood and organs, thereby improving prognosis.

The main limitation of our study is that the experiment failed to explore the specific mechanism by which statins increase intestinal inflammation, whether through the accumulation of harmful substances or activation of the immune system by microorganisms. Second, we failed to discover whether intestinal microbes or intestinal metabolites directly regulate the coagulation system, which requires more in-depth mechanistic studies in the future. Third, while all the animals in this study had free access to food and water throughout the experiment, it is possible that oral intake decreased after the onset of endotoxin 
exposure. The experiments did not examine the role of nutrition in intestinal hyperpermeability, and starvation itself can induce gut barrier dysfunction.

\section{Conclusions}

We found that simvastatin attenuates DIC by enhancing anticoagulation and fibrinolysis, accompanied by a decrease in organ microthrombus and a reduction in tissue damage, thereby prolonging survival. The intestine plays an important role in this process. Simvastatin pretreatment increases TJ protein and antimicrobial peptide expression and reduces intestinal permeability. Changes in the intestinal flora in the simvastatin group included elevated Lactobacillales and reduced Desulfovibrio and Mucispirillum, which might be related to prognosis. In addition, simvastatin reduced intestinal permeability, thereby reducing translocation of intestinal flora to organs and blood and avoiding the aggravation of primary infection due to intestinal disorders. In conclusion, simvastatin improves the prognosis of DIC by regulating the intestinal microenvironment and has the potential to improve the survival of clinical patients.

\section{List Of Abbreviations}

DIC: Disseminated intravascular coagulation; LPS: Lipopolysaccharide; PT: Prothrombin time; APTT: Activated partial thromboplastin time; PAI-1: Plasminogen activator inhibitor-1; ZO-1: Zonula occludens-1; JAM-A: Junction adhesion molecule; MODS: Multiple organ dysfunction syndrome; SIRS: systemic inflammatory response syndrome; TF: Tissue factor; NO: nitric oxide; FDP: Fibrinogen degradation products; ATIII: Antithrombin III activity; PLT: Platelet counts; AST: Aspartate aminotransferase; ALT: Alanine aminotransferase; BUN: Urea nitrogen; Cr: Creatinine; IL-6: Interleukin-6; IL-10: Interleukin-10; MCP1: Monocyte chemoattractant protein-1; IFN-y: Interferon- $\gamma$; TNF-a: Tumor necrosis factor-a; IL-1 $\beta$ : Interleukin-1 $\beta$; IL-18: Interleukin-18; ELISA: Enzyme-linked immunosorbent assays; TM: Thrombomodulin; TAT: Thrombin-antithrombin complex; OTU: Operational taxonomic units; PCA: Principal coordinate analysis; C3gnt: Core 3ß1, 3-N-acetyl glucosaminyltransferase; IAP: Intestinal alkaline phosphatase; MLCK: Myosin light chain kinase.

\section{Declarations}

\section{Ethics approval and consent to participate[}

All animal studies were approved by the Animal Experimentation Ethics Committee of Huazhong University of Science and Technology. The Institutional Animal Care and Use Committee Number is 2266.

Consent for publication: Not applicable.

Availability of data and materials: All data generated or analysed during this study are included in this published article [and its supplementary information files]. 
Competing interests: This manuscript has been read and approved by all authors, and the authors state that they have no conflicts of interest.

Funding: This study was supported by the National Natural Science Foundation of China (Nos. 81873434 and 81570116 to Heng Mei).

Authors' contributions: Min Xu and Heng Mei designed the study, analysed the data and wrote the paper; Min Xu, Lili Luo and Mengyi Du performed the experiments; Lu Tang, Jie Zhou and Yu Hu analysed the data and modified the grammatical structure of the article; all authors reviewed the paper and approved the final manuscript.

Acknowledgements: The authors would like to thank all the study participants and the Thrombosis and Hemostasis Laboratory of Hubei Provincial.

\section{References}

1. Levi M, Ten Cate H: Disseminated Intravascular Coagulation - NEJM. Journal of Thrombosis \& Haemostasis 2010, 8:1475-1476.

2. Levi M, Dörffler-Melly J, Reitsma P, Buller H, Florquin S, van der Poll T, Carmeliet P: Aggravation of endotoxin-induced disseminated intravascular coagulation and cytokine activation in heterozygous protein-C-deficient mice. Blood 2003, 101:4823-4827.

3. Gando S, Shiraishi A, Yamakawa K, Ogura H, Saitoh D, Fujishima S, Mayumi T, Kushimoto S, Abe T, Shiino Y, et al: Role of disseminated intravascular coagulation in severe sepsis. Thromb Res 2019, 178:182-188.

4. Rangel-Frausto MS, Pittet D, Costigan M, Hwang T, Davis CS, Wenzel RP: The natural history of the systemic inflammatory response syndrome (SIRS). A prospective study. Jama 1995, 273:117-123.

5. Fay KT, Ford ML, Coopersmith CM: The intestinal microenvironment in sepsis. Biochim Biophys Acta Mol Basis Dis 2017, 1863:2574-2583.

6. Kumar M, Leon Coria A, Cornick S, Petri B, Mayengbam S, Jijon HB, Moreau F, Shearer J, Chadee K: Increased intestinal permeability exacerbates sepsis through reduced hepatic SCD-1 activity and dysregulated iron recycling. Nature communications 2020, 11:483.

7. Shimizu K, Ogura H, Goto M, Asahara T, Nomoto K, Morotomi M, Yoshiya K, Matsushima A, Sumi Y, Kuwagata Y, et al: Altered gut flora and environment in patients with severe SIRS. J Trauma 2006, 60:126-133.

8. MacFie J, O'Boyle C, Mitchell CJ, Buckley PM, Johnstone D, Sudworth P: Gut origin of sepsis: a prospective study investigating associations between bacterial translocation, gastric microflora, and septic morbidity. Gut 1999, 45:223-228.

9. Deitch EA: Bacterial translocation or lymphatic drainage of toxic products from the gut: what is important in human beings? Surgery 2002, 131:241-244. 
10. Arnaud H, Fabien L, Vanina L, Pierre M: Simvastatin inhibits the pro-inflammatory and pro-thrombotic effects of IL-17 and TNF-a on endothelial cells. Annals of the Rheumatic Diseases 2013, 72:754-760.

11. Beigel Y, Fuchs J, Snir M, Green P, Lurie Y, Djaldetti M: Lovastatin therapy in hypercholesterolemia: effect on fibrinogen, hemorrheologic parameters, platelet activity, and red blood cell morphology. $J$ Clin Pharmacol 1991, 31:512-517.

12. Mortensen EM, Restrepo MI, Copeland LA, Pugh JA, Anzueto A, Cornell JE, Pugh MJ: Impact of previous statin and angiotensin II receptor blocker use on mortality in patients hospitalized with sepsis. Pharmacotherapy 2007, 27:1619-1626.

13. Merx MW, Liehn EA, Janssens U, Lütticken R, Schrader J, Hanrath P, Weber C: HMG-CoA reductase inhibitor simvastatin profoundly improves survival in a murine model of sepsis. Circulation 2004, 109:2560-2565.

14. Sahebkar A, Catena C, Ray KK, Vallejo-Vaz AJ, Reiner Ž, Sechi LA, Colussi G: Impact of statin therapy on plasma levels of plasminogen activator inhibitor-1. A systematic review and meta-analysis of randomised controlled trials. Thromb Haemost 2016, 116:162-171.

15. Eto M, Kozai T, Cosentino F, Joch H, Lüscher TF: Statin prevents tissue factor expression in human endothelial cells: role of Rho/Rho-kinase and Akt pathways. Circulation 2002, 105:1756-1759.

16. Han X, Fink MP, Delude RL: Proinflammatory cytokines cause NO*-dependent and -independent changes in expression and localization of tight junction proteins in intestinal epithelial cells. Shock 2003, 19:229-237.

17. Han X, Fink MP, Yang R, Delude RL: Increased iNOS activity is essential for intestinal epithelial tight junction dysfunction in endotoxemic mice. Shock 2004, 21:261-270.

18. Gong X, Ma Y, Ruan Y, Fu G, Wu S: Long-term atorvastatin improves age-related endothelial dysfunction by ameliorating oxidative stress and normalizing eNOS/iNOS imbalance in rat aorta. Exp Gerontol 2014, 52:9-17.

19. Peng H, Luo P, Li Y, Wang C, Liu X, Ye Z, Li C, Lou T: Simvastatin alleviates hyperpermeability of glomerular endothelial cells in early-stage diabetic nephropathy by inhibition of RhoA/ROCK1. PLOS ONE 2013, 8:e80009.

20. Caparrós-Martín JA, Lareu RR, Ramsay JP, Peplies J, Reen FJ, Headlam HA, Ward NC, Croft KD, Newsholme P, Hughes JD, O'Gara F: Statin therapy causes gut dysbiosis in mice through a PXRdependent mechanism. Microbiome 2017, 5:95.

21. Sun B, Li L, Zhou X: Comparative analysis of the gut microbiota in distinct statin response patients in East China. Journal of microbiology (Seoul, Korea) 2018, 56:886-892.

22. He X, Zheng N, He J, Liu C, Feng J, Jia W, Li H: Gut Microbiota Modulation Attenuated the Hypolipidemic Effect of Simvastatin in High-Fat/Cholesterol-Diet Fed Mice. J Proteome Res 2017, 16:1900-1910.

23. Cani PD, Bibiloni R, Knauf C, Waget A, Neyrinck AM, Delzenne NM, Burcelin R: Changes in gut microbiota control metabolic endotoxemia-induced inflammation in high-fat diet-induced obesity and diabetes in mice. Diabetes 2008, 57:1470-1481. 
24. Huse SM, Welch DM, Morrison HG, Sogin ML: Ironing out the wrinkles in the rare biosphere through improved OTU clustering. Environ Microbio/ 2010, 12:1889-1898.

25. Coopersmith CM, Stromberg PE, Dunne WM, Davis CG, Nd AD, Buchman TG, Karl IE, Hotchkiss RS: Inhibition of intestinal epithelial apoptosis and survival in a murine model of pneumonia-induced sepsis. Jama 2002, 287:1716.

26. Zhang-Sun W, Augusto LA, Zhao L, Caroff M: Desulfovibrio desulfuricans isolates from the gut of a single individual: structural and biological lipid A characterization. FEBS Lett 2015, 589:165-171.

27. Belzer C, Gerber GK, Roeselers G, Delaney M, DuBois A, Liu Q, Belavusava V, Yeliseyev V, Houseman A, Onderdonk A, et al: Dynamics of the microbiota in response to host infection. PLOS ONE 2014, 9:e95534.

28. Bergstrom KS, Kissoon-Singh V, Gibson DL, Ma C, Montero M, Sham HP, Ryz N, Huang T, Velcich A, Finlay BB, et al: Muc2 protects against lethal infectious colitis by disassociating pathogenic and commensal bacteria from the colonic mucosa. PLoS Pathog 2010, 6:e1000902.

29. Xia L: Core 3-derived O-glycans are essential for intestinal mucus barrier function. Meth Enzymol 2010, 479:123-141.

30. Franco RF, de Jonge E, Dekkers PE, Timmerman JJ, Spek CA, van Deventer SJ, van Deursen P, van Kerkhoff $L$, van Gemen $B$, ten Cate $H$, et al: The in vivo kinetics of tissue factor messenger RNA expression during human endotoxemia: relationship with activation of coagulation. Blood 2000, 96:554-559.

31. Pathak R, Ghosh SP, Zhou D, Hauer-Jensen M: The Vitamin E Analog Gamma-Tocotrienol (GT3) and Statins Synergistically Up-Regulate Endothelial Thrombomodulin (TM). International journal of molecular sciences 2016, 17.

32. Panigrahi P, Parida S, Nanda NC, Satpathy R, Pradhan L, Chandel DS, Baccaglini L, Mohapatra A, Mohapatra SS, Misra PR, et al: A randomized synbiotic trial to prevent sepsis among infants in rural India. Nature 2017, 548:407-412.

33. Shimizu K, Yamada T, Ogura H, Mohri T, Kiguchi T, Fujimi S, Asahara T, Yamada T, Ojima M, Ikeda M, Shimazu T: Synbiotics modulate gut microbiota and reduce enteritis and ventilator-associated pneumonia in patients with sepsis: a randomized controlled trial. Crit Care 2018, 22:239.

34. Farhadi A, Keshavarzian A, Ranjbaran Z, Fields JZ, Banan A: The role of protein kinase $C$ isoforms in modulating injury and repair of the intestinal barrier. J Pharmacol Exp Ther 2006, 316:1-7.

35. Scott KG, Meddings JB, Kirk DR, Lees-Miller SP, Buret AG: Intestinal infection with Giardia spp. reduces epithelial barrier function in a myosin light chain kinase-dependent fashion. Gastroenterology 2002, 123:1179-1190.

36. Trivedi K, Barrett KE, Resta-Lenert SC: Probiotic inhibition of the entry of enteroinvasive E. coli into, human intestinal epithelial cells involves both Rho-dependent and -independent pathways. Gastroenterology 2003, 124:A106-A106.

37. Takada K, Ohno N, Yadomae T: Detoxification of lipopolysaccharide (LPS) by egg white lysozyme. FEMS Immunol Med Microbiol 1994, 9:255-263. 
38. Estaki M, DeCoffe D, Gibson DL: Interplay between intestinal alkaline phosphatase, diet, gut microbes and immunity. World J Gastroenterol 2014, 20:15650-15656.

39. Vaishnava S, Yamamoto M, Severson KM, Ruhn KA, Yu X, Koren O, Ley R, Wakeland EK, Hooper LV: The antibacterial lectin Regllgamma promotes the spatial segregation of microbiota and host in the intestine. Science 2011, 334:255-258.

40. Nolan JA, Skuse P, Govindarajan K, Patterson E, Konstantinidou N, Casey PG, MacSharry J, Shanahan F, Stanton C, Hill C, et al: The influence of rosuvastatin on the gastrointestinal microbiota and host gene expression profiles. Am J Physiol Gastrointest Liver Physio/ 2017, 312:G488-g497.

41. Cekanaviciute E, Yoo BB, Runia TF, Debelius JW, Singh S, Nelson CA, Kanner R, Bencosme Y, Lee YK, Hauser SL, et al: Gut bacteria from multiple sclerosis patients modulate human T cells and exacerbate symptoms in mouse models. Proc Natl Acad Sci USA 2017, 114:10713-10718.

\section{Figures}




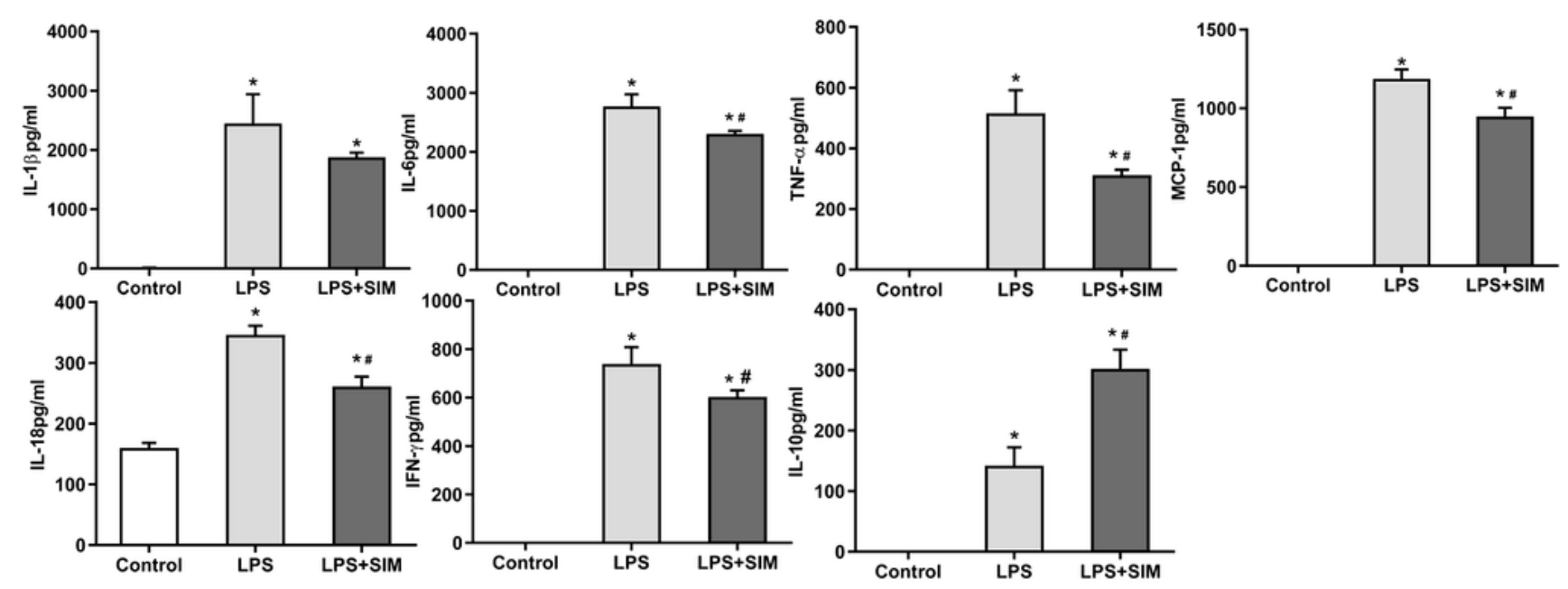

B.Lung

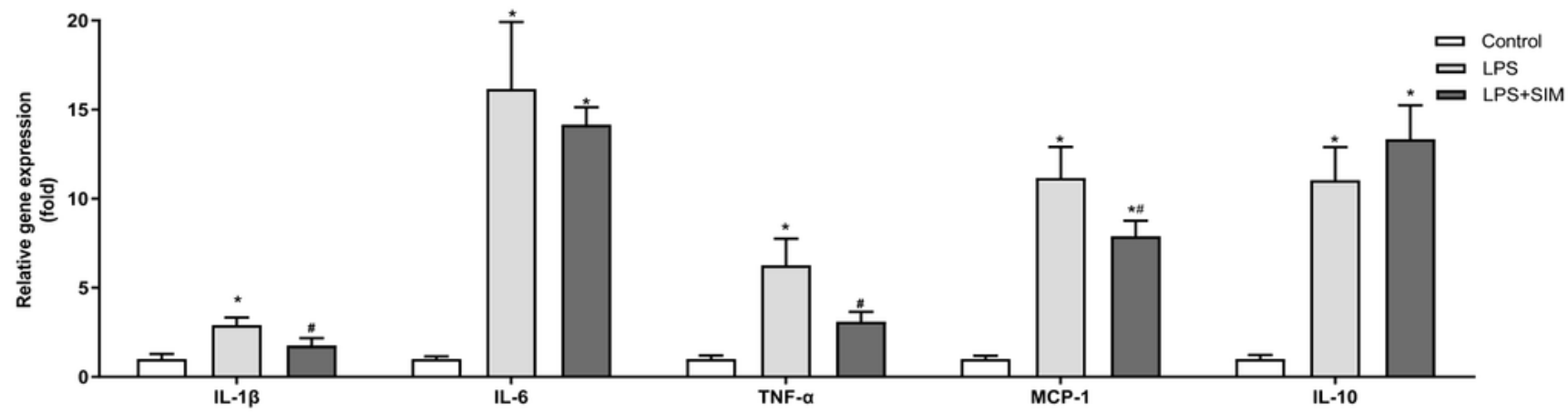

C.Lung
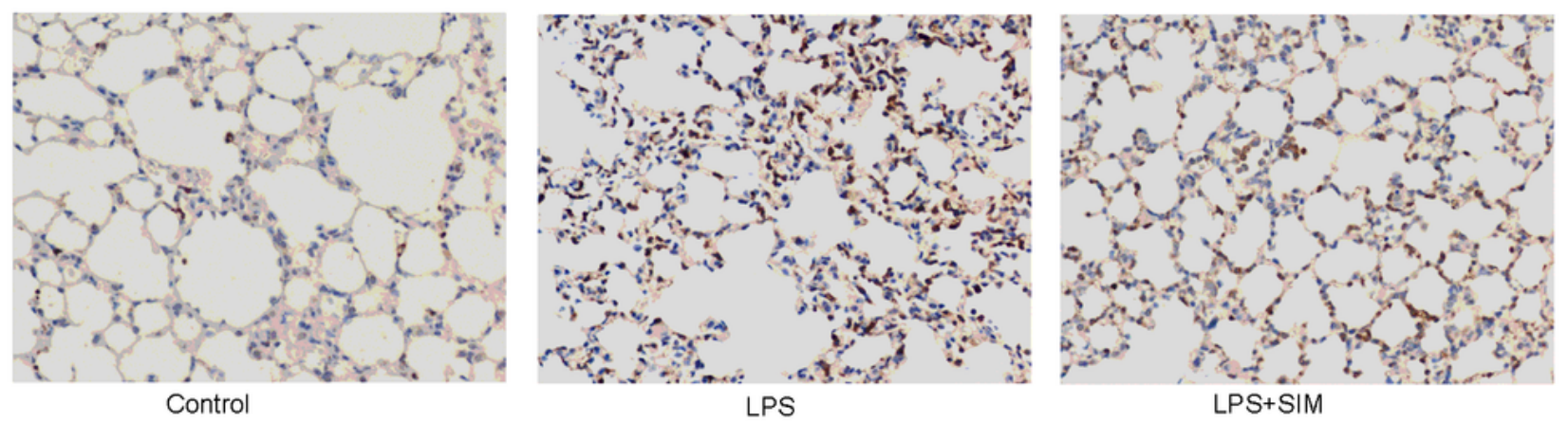

D.Plasma
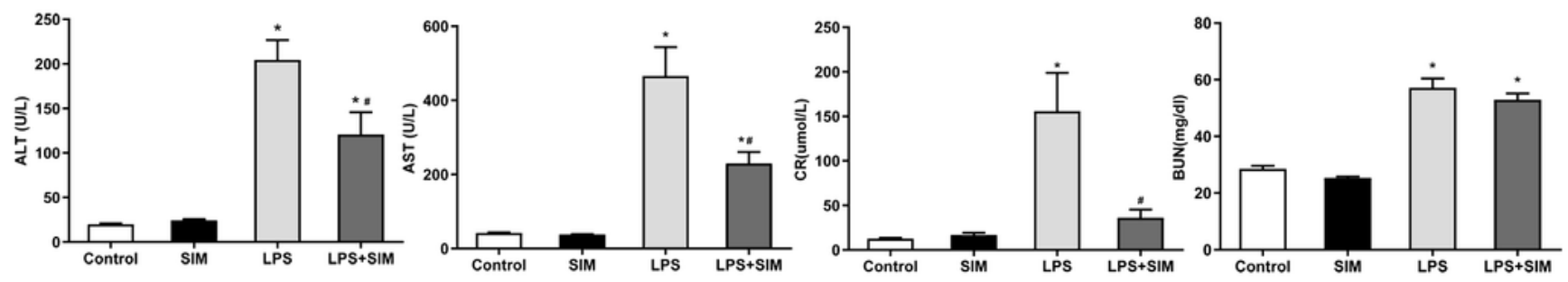

\section{Figure 1}

Simvastatin pretreatment attenuates LPS-induced inflammatory responses and organ damage in mice at $12 \mathrm{~h}$. (A) The plasma levels of inflammatory factors in the indicated groups were measured using ELISAs or a Mouse Inflammation CBA kit. (B) The mRNA levels of the inflammatory factors in lungs were detected by qPCR. (C) Anti-granulocyte staining of lung tissues. Representative images (magnification, 10x; inset, 40x) are shown. (D) Plasma levels of ALT, AST, CR and BUN in the indicated groups. The data 
are presented as the mean \pm SEM $(n=8)$. ${ }^{*} \mathrm{P}<0.05$ compared with the control. \#P<0.05 comparisons between LPS group and LPS+SIM group.

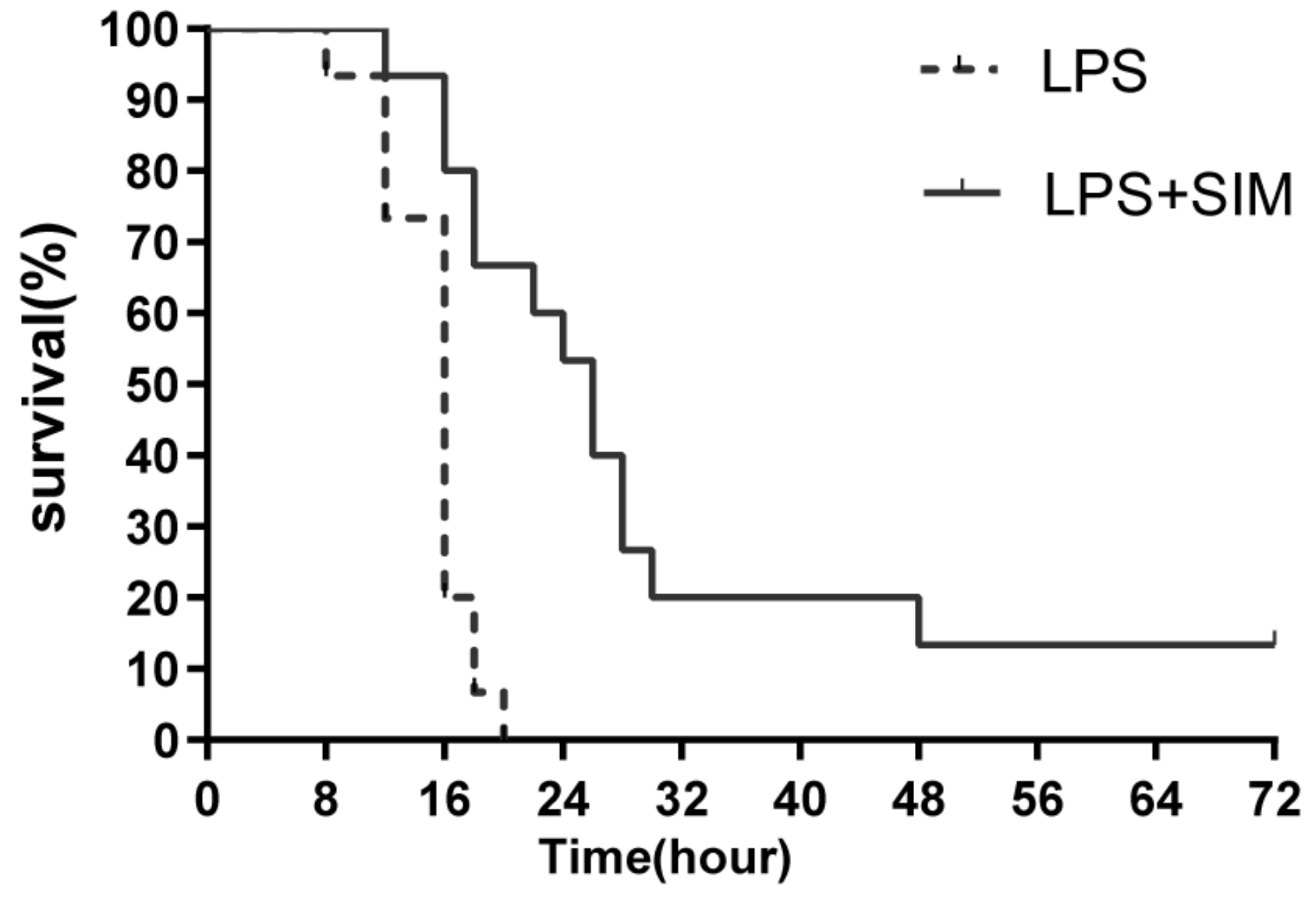

Figure 2

Survival curves. The survival time of simvastatin-pretreated mice (broken line) was profoundly increased compared to that of untreated mice after endotoxin administration. No deaths occurred in the control group $(P<0.001, n=15)$. 

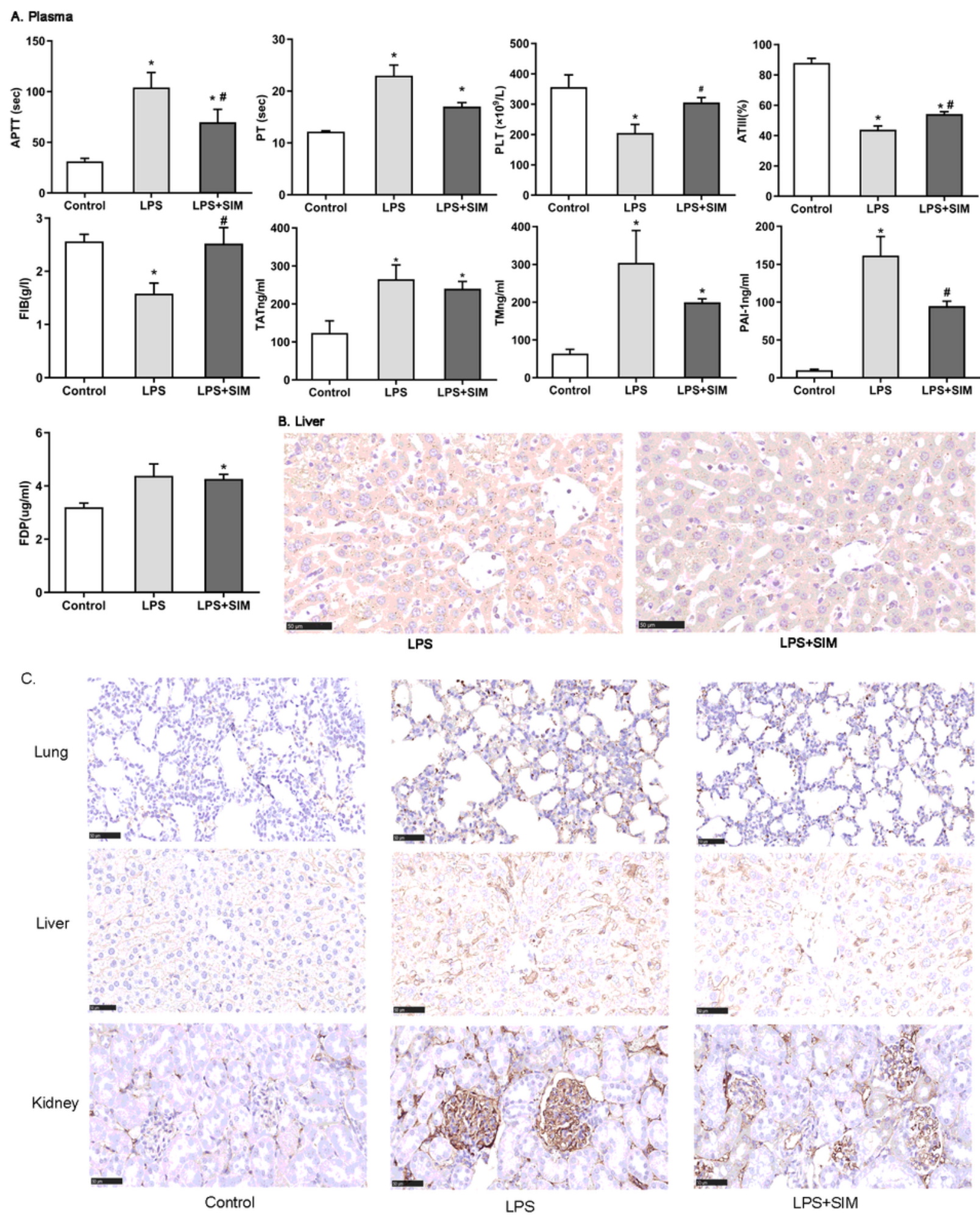

\section{Figure 3}

Simvastatin improves LPS-induced blood coagulation disorders and fibrin deposition in mice at $12 \mathrm{~h}$. (A) The PT, APTT, fibrinogen level, FDP level and ATIII activity were measured with an automated blood coagulation analyser; PLT was measured on an automated animal blood counter; and the plasma levels of TAT, PAl-1 and TM were measured using ELISA kits. (B) Immunohistochemical analysis of TF expression in the indicated group. Representative images (magnification, 10x; inset, 40x) are shown. (C) 
Fibrin staining of the lungs, kidneys and liver in each group. The data are presented as the mean \pm SEM $(n=7) .{ }^{*}<0.05$ compared with the control. \#P<0.05 comparisons between LPS group and LPS+SIM group.
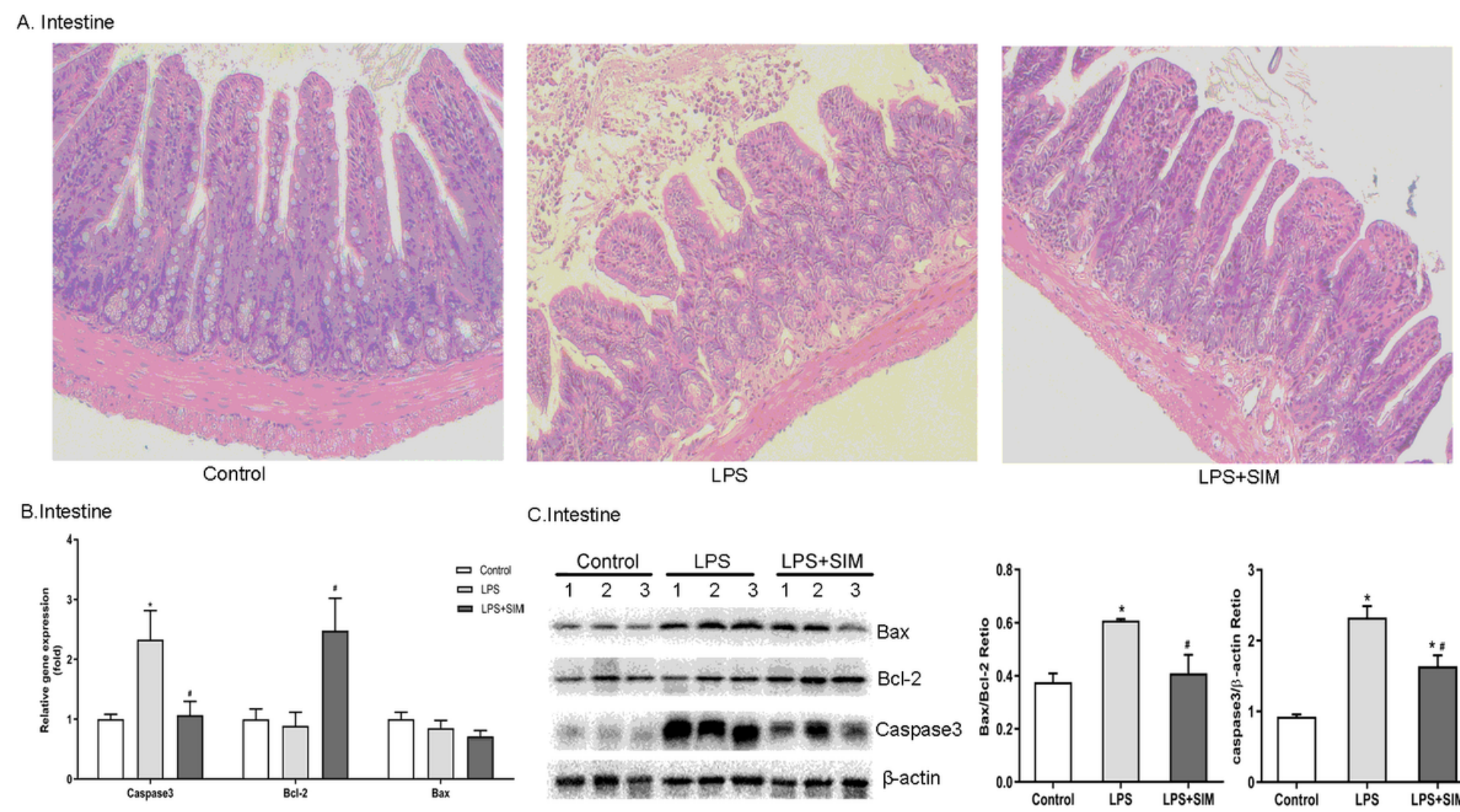

C.Intestine
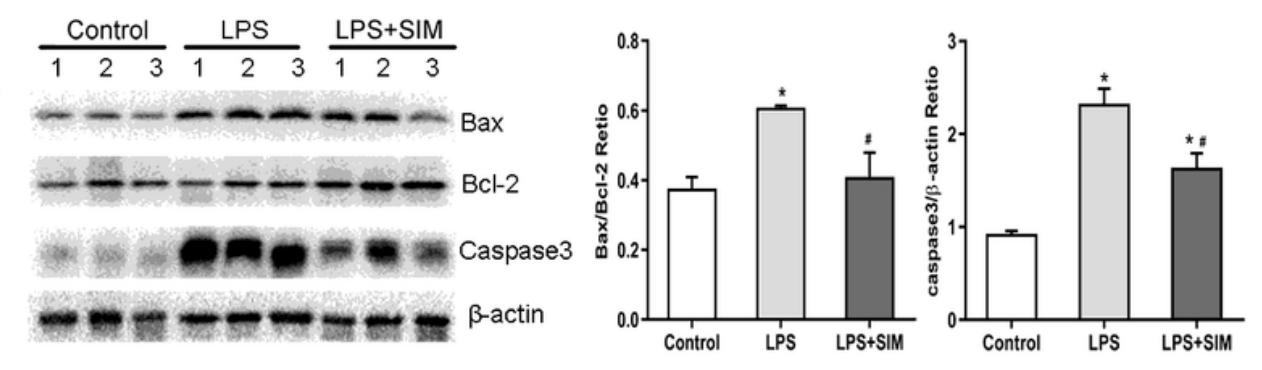

\section{Figure 4}

Simvastatin alleviates LPS-induced histological damage and apoptosis in the intestine at $12 \mathrm{~h}$. (A) Haematoxylin and eosin staining of ileal segments in the indicated groups (magnification, 10x; inset, 40x). (B) Western blotting and qPCR were used to determine the expression of caspase3, Bcl-2 and Bax at the protein level (right) and mRNA level (left). The data are presented as the mean \pm SEM $(n=8) .{ }^{*}<0.05$ compared with the control. \#P $<0.05$ comparisons between LPS group and LPS+SIM group. 
A

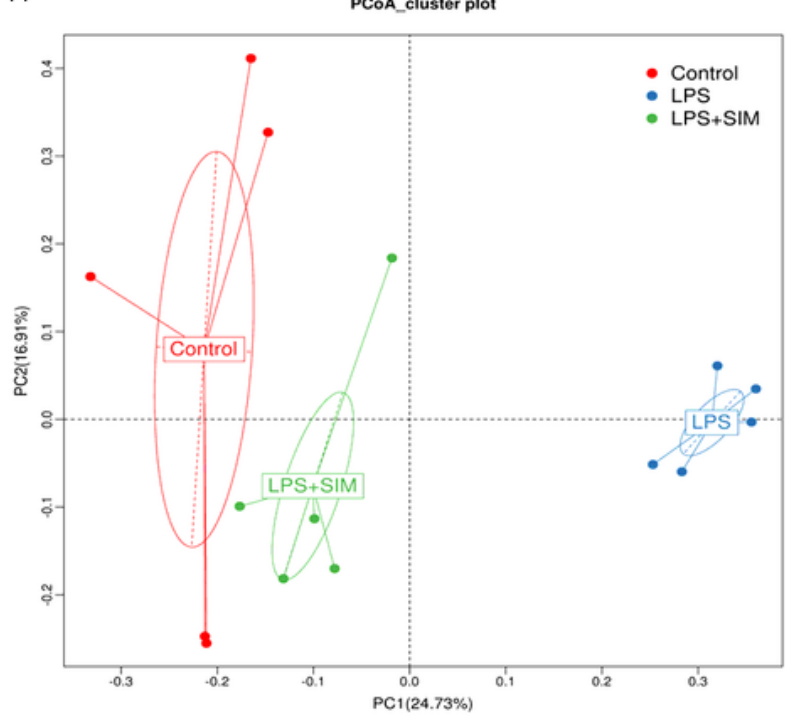

C

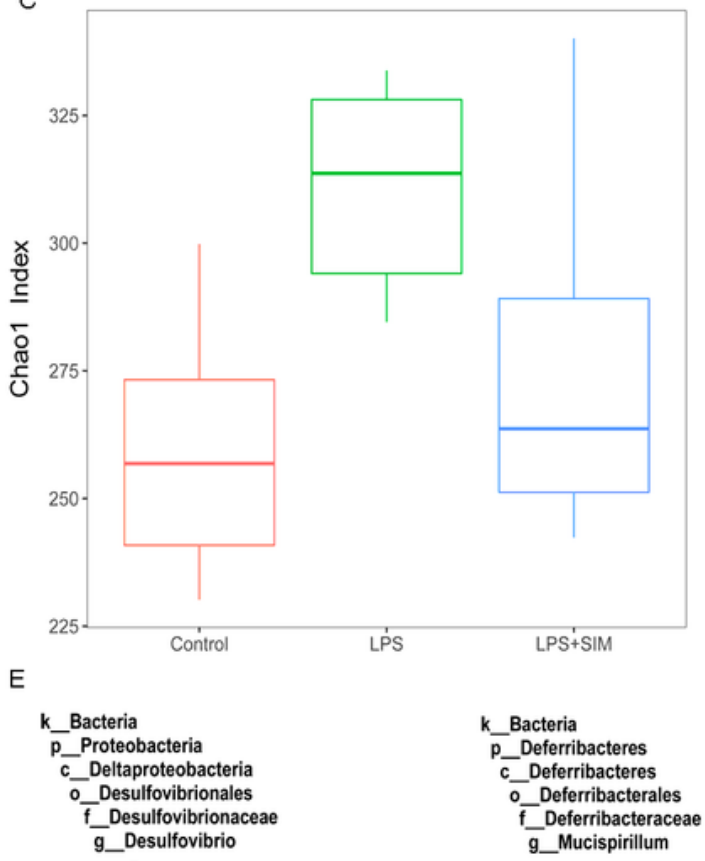

B
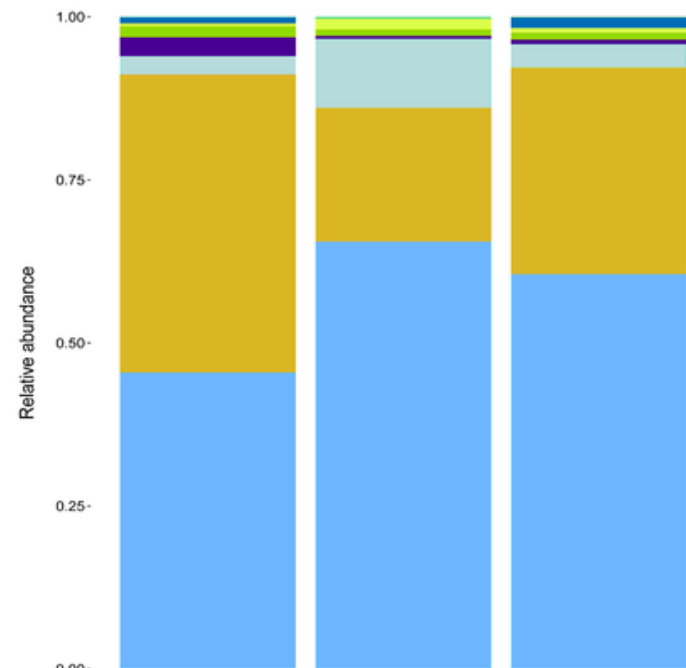

Phylum

Cyanobacteria Verrucomicrobia Patescibacteria Actinobacteria Proteobacteria Bacteroidetes
D

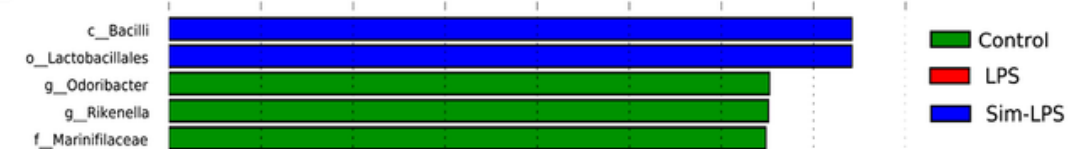

P_Actinobacteria

C_Coriobacteriia

f_Eggerthellaceae

9_Enterorhabdus

f_Ruminococcaceae

9_Desulfovibrio

c_Deltaproteobacteria

Desulfovibrionaceae

-_Desulfovibrionales

9_lleibacterium

_Deferribacteraceae

-_Deferribacterales

D_Deferribacteres

C_Deferribacteres

9_Mucispirillum

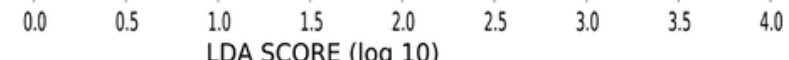

LDA SCORE $(\log 10)$
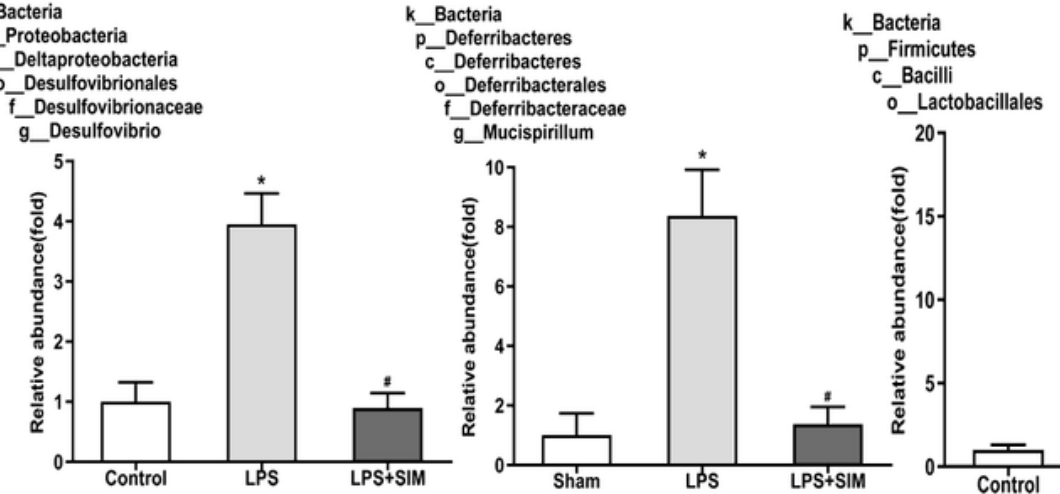

k_Bacteria

P_Firmicutes

_Clostridiales

_Lactobacillales
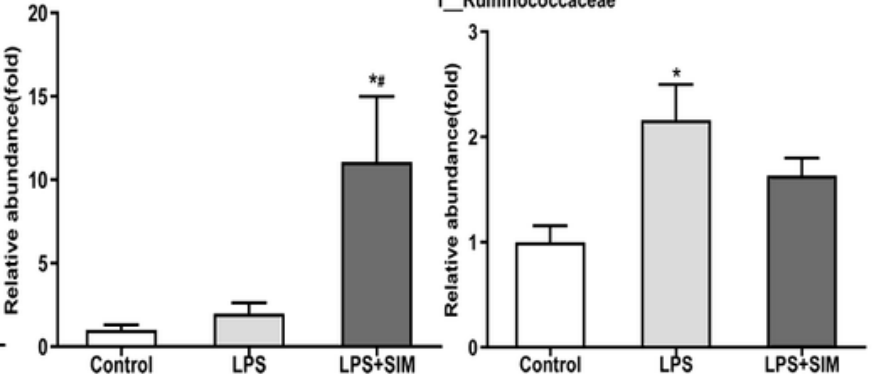

\section{Figure 5}

Simvastatin-pretreated mice show different gut microbiota compositions. (A) PCA score plot. (B) Gut microbiota distribution at the phylum level. (C) Chao1 index. (D) Distinctive gut microbiota composition revealed by linear discriminant analysis (LDA) in the indicated groups. (E) Comparison of relative taxonomic abundance among the indicated groups $(n=5)$. ${ }^{*}<<0.05$ compared with the control. \# $P<0.05$ comparisons between LPS group and LPS+SIM group. 


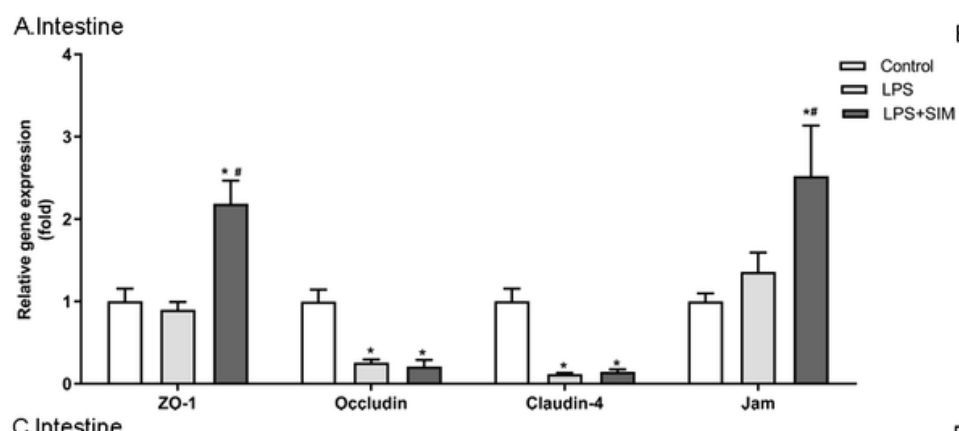

B.Caco-2
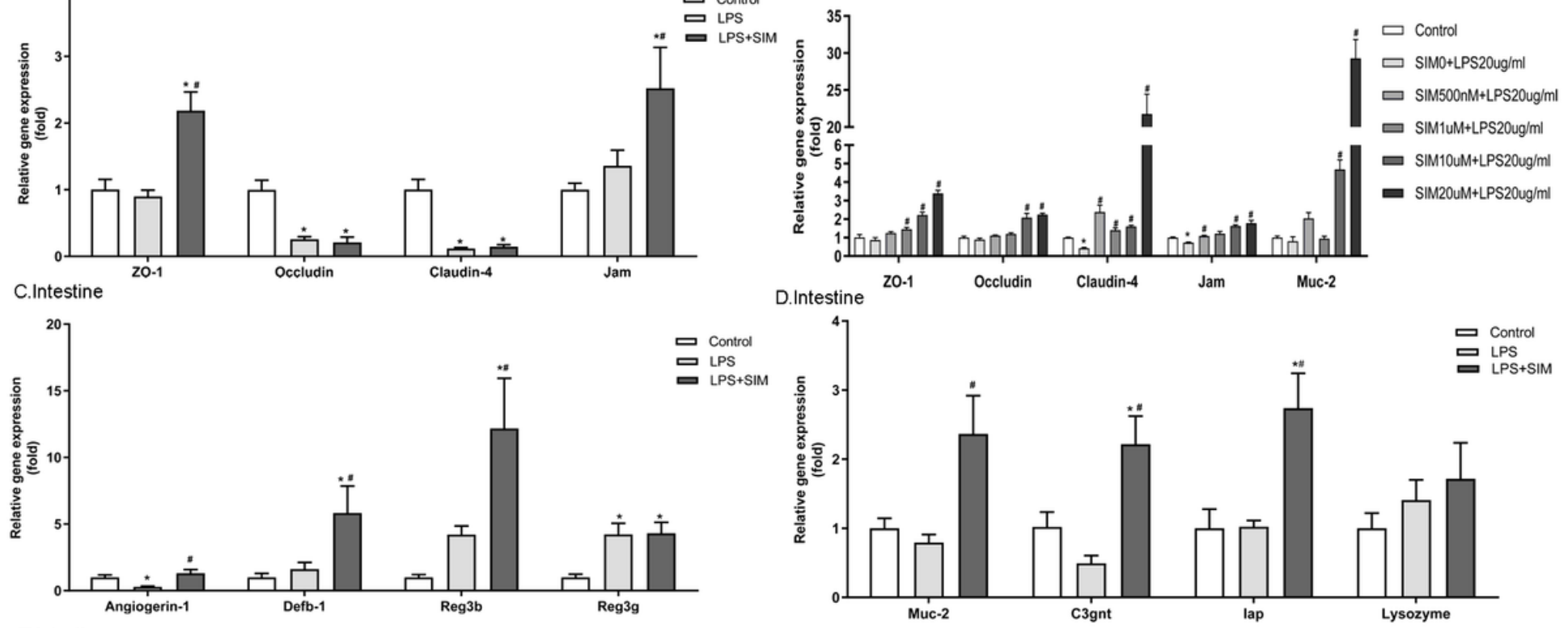

E.Intestine
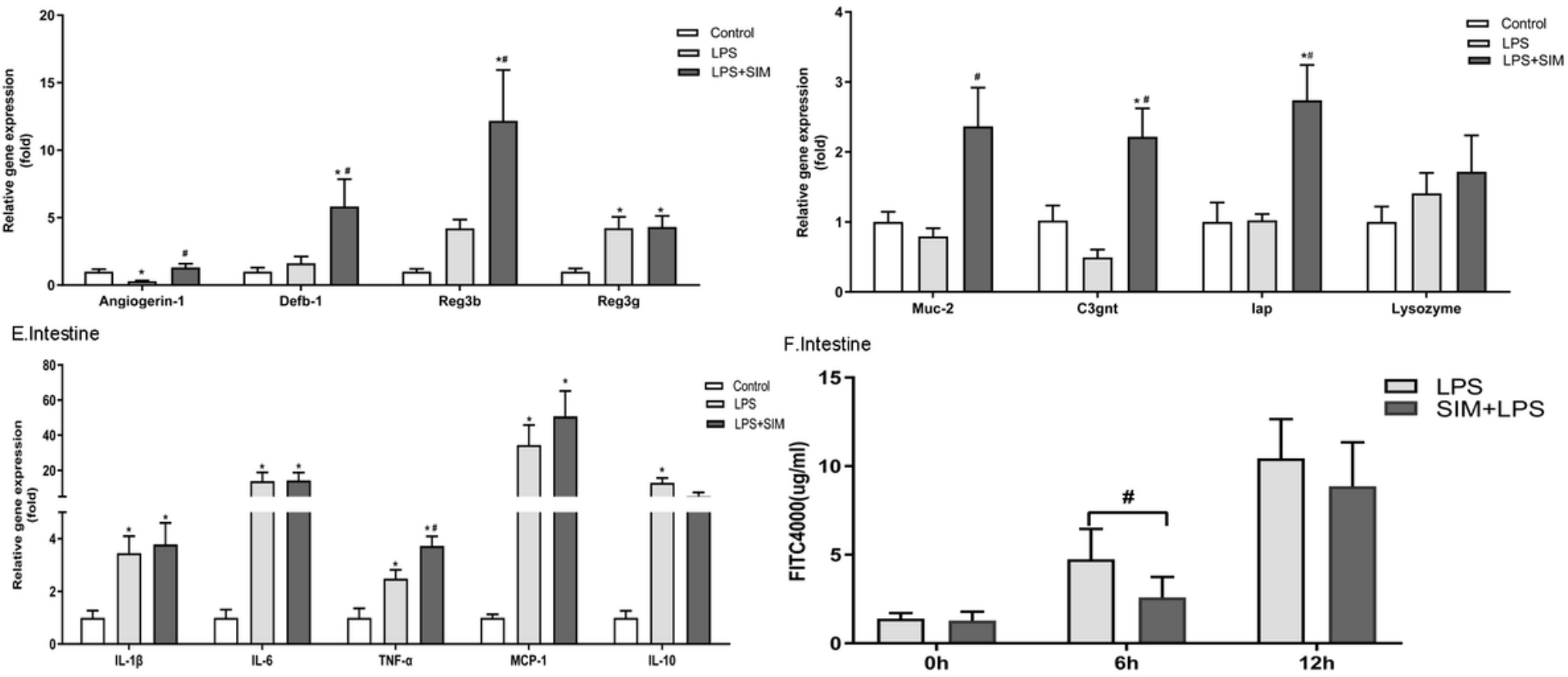

F.Intestine

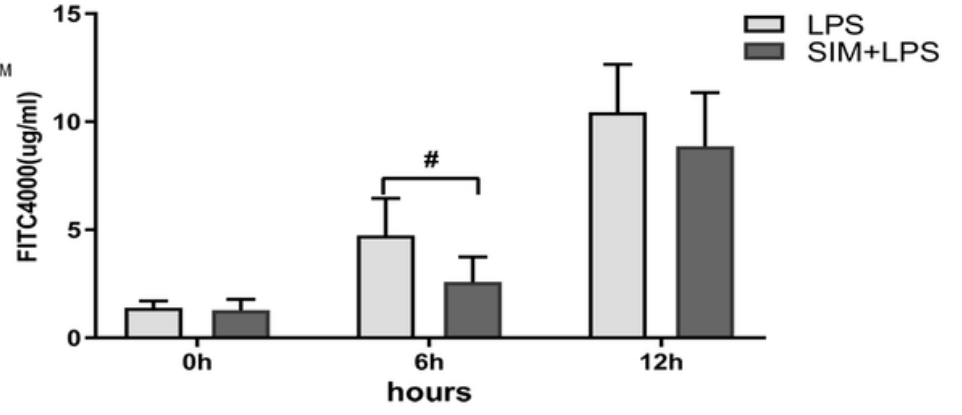

Figure 6

Simvastatin pretreatment improves intestinal barrier function. The mRNA levels of (A) TJ components, (C) antibacterial peptides, (D) mucin and (E) inflammatory cytokines in the ileum of the indicated groups. (B) The mRNA levels of TJ components in Caco-2 cells. (F) FITC-Dextran (FD-4) levels in plasma. The data are presented as the mean \pm SEM $(n=8)$. ${ }^{*}<0.05$ compared with the control. \# $P<0.05$ comparisons between LPS group and LPS+SIM group. 

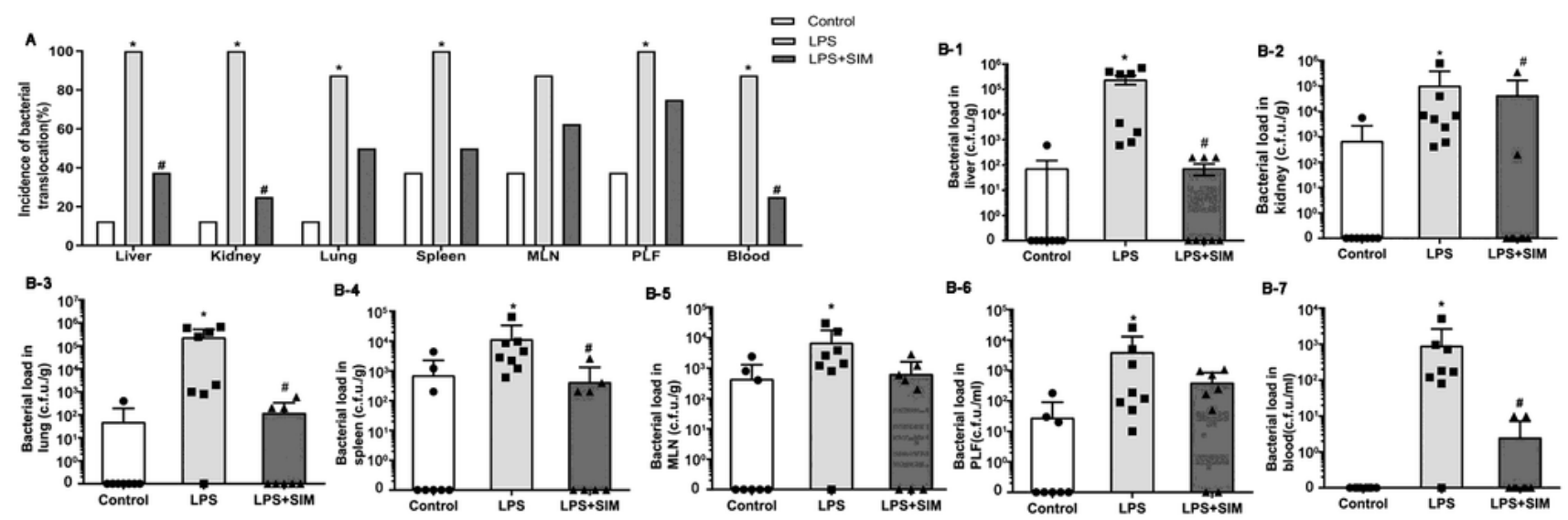

D
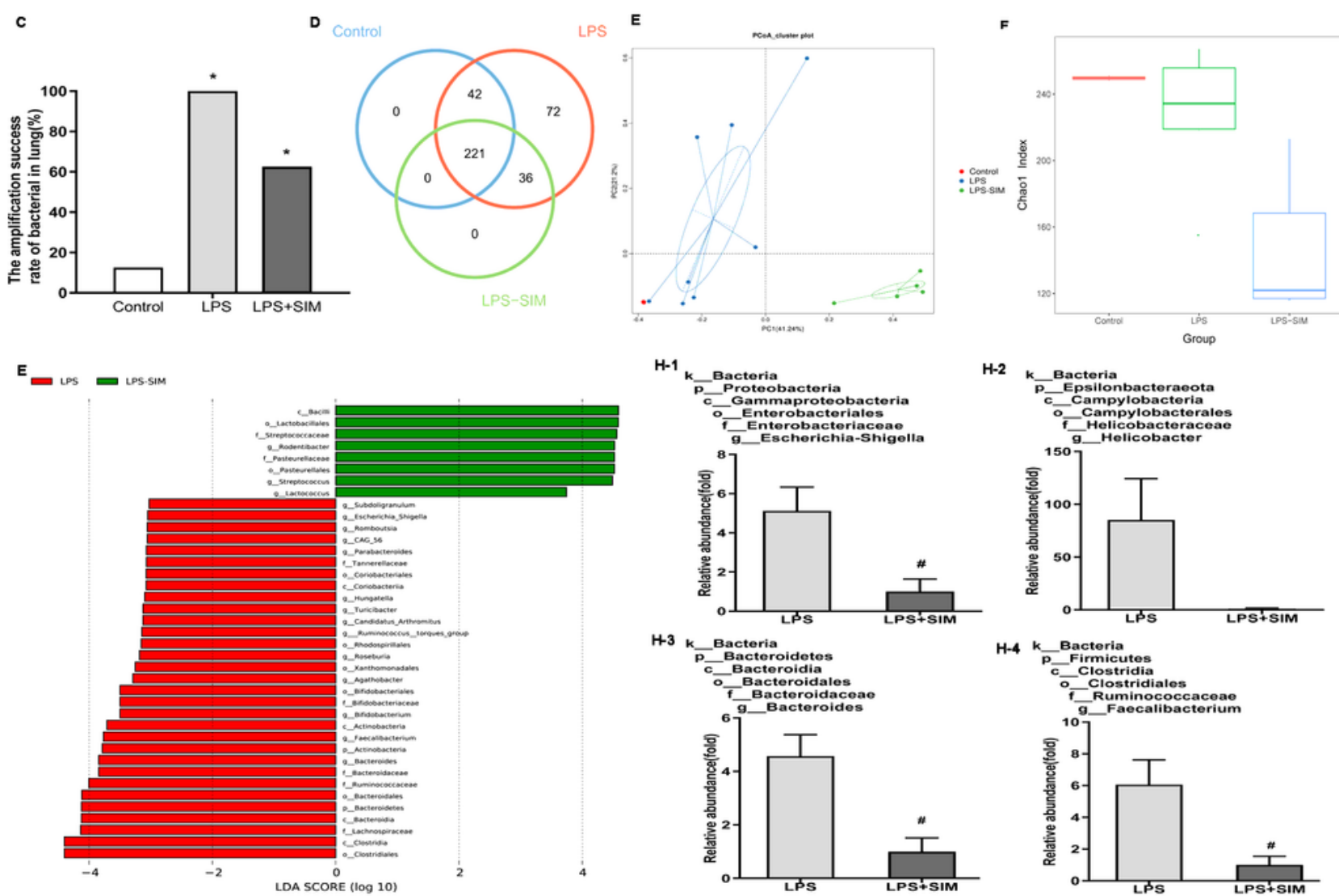

H-2
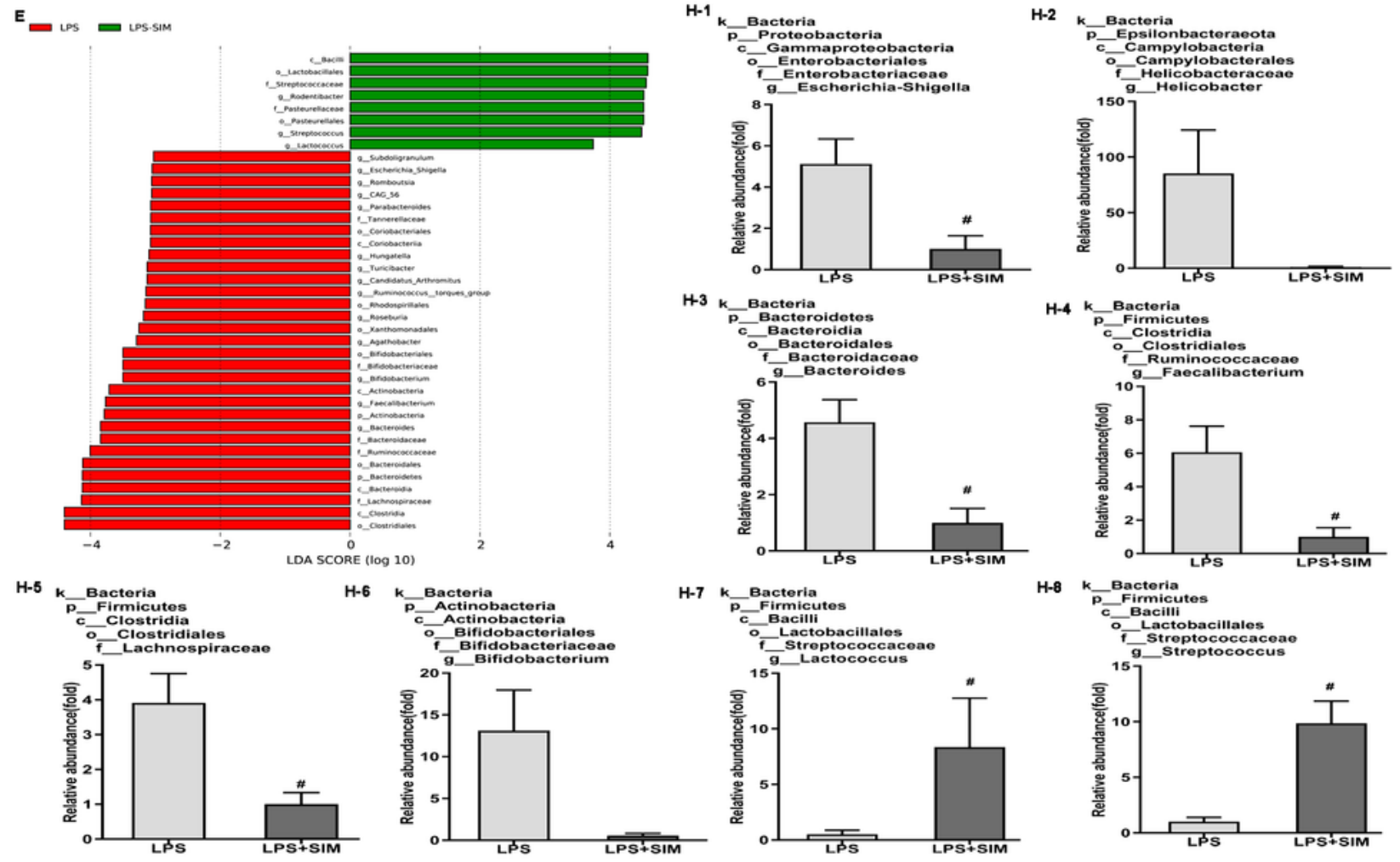

Figure 7

Simvastatin inhibits bacterial translocation. (A) Incidence of bacterial translocation (\%). (B1-8) Bacterial load (c.f.u./g) in the liver, kidney, lung, spleen, blood, mesenteric lymph nodes (MLNs) and peritoneal lavage fluid (PLF). (C) The amplification success rate of bacteria in lungs. (D) Venn diagram; (E) PCoA score plot; (F) Chao1 index. (G) Distinctive gut microbiota composition revealed by linear discriminant analysis (LDA) in the indicated groups. (H) Comparison of relative taxonomic abundance among the 
indicated groups. The data are presented as the mean \pm SEM $(n=8) .{ }^{*} P<0.05$ compared with the control. \# $\mathrm{P}<0.05$ comparisons between LPS group and LPS+SIM group.

\section{Supplementary Files}

This is a list of supplementary files associated with this preprint. Click to download.

- SupplementTable1.doc 